\title{
Are Retrospective Patient Chart Audits an Affordable and Reliable Answer to Healthcare Data Needs? Assessing the Ground Reality
}

\author{
Uttam Barick*1, Anto Vijaykanth ${ }^{1}$, Hitesh Bharucha ${ }^{1}$, Arun Gowda ${ }^{1}$, Anand Patil ${ }^{1}$, Stefan Bosbach ${ }^{2}$ and Behsad \\ Zomorodi $^{2}$ \\ ${ }^{1}$ phamax Analytic Resources, India \\ ${ }^{2}$ phamax AG, Switzerland
}

Received: 盋 July 12, 2018; Published: 制July 25, 2018

*Corresponding author: Uttam Barick, phamax Analytic Resources Pvt. Ltd. \#19, KMJ Ascend, $1^{\text {st }}$ Cross, $17^{\text {th }}$ C Main, $5^{\text {th }}$ Block, Koramangala, Bangalore, India

\begin{abstract}
Medical records at various institutes and hospitals are unrecognized and not utilized to the fullest in spite of their relevance in identifying crucial information on patient and disease management. If this medical data is appropriately channelized by outcome driven analysis plans like retrospective chart audits, the results will certainly provide insights for many unanswered medical queries. Retrospective chart audits are the scientific and systematic review of existing medical records suited for the quality assessments of patients with lifestyle disorders (like Obesity, Diabetes, etc.), specifically in epidemiology research. They also assist researchers to understand the safety profile of certain classes of drugs (e.g. Psychiatry and CNS disorders). This article offers an overview on the applications and the business insights derived from retrospective chart audits whereby assessing the reality of implementing this methodology in terms of standard clinical research and regulatory requirements.

Keywords: Retrospective Patient Chart Audit; Electronic Medical Records; Real World Evidence; Healthcare Data
\end{abstract}

\section{Introduction}

Medical records or charts are valuable resources for essential clinical information. A retrospective chart review (RCR) or medical chart audit (MCA) is a well-established research design in which pre-recorded medical or clinical information in the form of charts are thoroughly reviewed to arrive at research and business insights [1]. Retrospective chart audits are neglected and largely undervalued compared to randomized clinical trials in spite of their capabilities to harness valuable insights from historical medical records [2]. They could potentially provide meaningful insights in relatively lesser time and on a limited budget [3]. Chart audits have several distinct advantages like the relatively inexpensive ability to explore rich readily accessible existing data-sets within less time, easier access to conditions with a long latency between exposure and disease, the ability to study rare occurrences, and most importantly, the generation of hypotheses that can be tested prospectively [2]. Though RCRs have many advantages, they also have some limitations, which restrict researchers from adopting them. However, the advancement of technology and the advent of Electronic Medical Records (EMR) have made life easier for researchers in overcoming these limitations effortlessly. Less than a decade ago, nine out of ten doctors in the US updated their patient files manually and retained them in colour coded files. A report says that at the end of 2017, approximately $90 \%$ physicians will use the EMR system [4]. This report itself outlines the future landscape of the data collection method. The EMR system was introduced some time back in some Nordic countries in Europe, and within very less time these countries have $100 \%$ penetration in terms of both primary and secondary data. The EMR adoption rate among various hospitals from 2012 to 2017 has increased significantly at the rate of $27 \%$ [5]. Considering that EMRs are better accepted and adopted by clinicians, they provide a promising option for tapping into hitherto unavailable data.

\section{Discussion}

Patient chart review studies were conventionally used to understand the burden of illness and patterns of care in hospitals or treatment institutes. However, this methodology is increasingly being adopted to cater to the data requirements associated with marketing authorization and risk management, which also includes the data on off-label medication use. Pharmaceutical companies are more enticed towards these types of studies considering the research benefits, since they help generate significant insights from hitherto under-utilized resources. 


\section{What is a Patient Chart Audit?}

A chart audit is a part of a retrospective assessment that unravels the potential of healthcare data. It evaluates a product's prescription choices and patterns of treatment algorithms to understand the effectiveness of the care given. Chart audits are widely used as a data collection method for studies on incidence, prevalence, clinical course, prognosis of specific conditions (clinical epidemiology), determinants/outcomes of health service use (health care epidemiology), retrospective data to answer clinical queries, adherence to guidelines, or standards of practice [1].

\section{Understanding the Current State of Art and Different Data Sources of a Chart Audit}

Chart audit data exists in many forms: electronic databases, results from diagnostictests, and notes from health service providers, to mention a few. A chart audit is a popular methodology widely applied in many healthcare-based disciplines such as epidemiology, quality assessment, professional education and residency training, inpatient care, and clinical research, and valuable information can be gathered from study results for subsequent prospective studies [2]. Chart audits serve many purposes, from compliance to research to administrative to clinical [6]. They can be conducted on virtually any aspect of care ordinarily documented in the medical record. Sometimes, clinical practitioners get puzzled by various clinical processes that do not operate as expected. In such cases, chart audits help to identify problems immediately and rectify them [7]. The most important use of a chart audit is to measure the quality of care so that it can be improved (Figure 1).

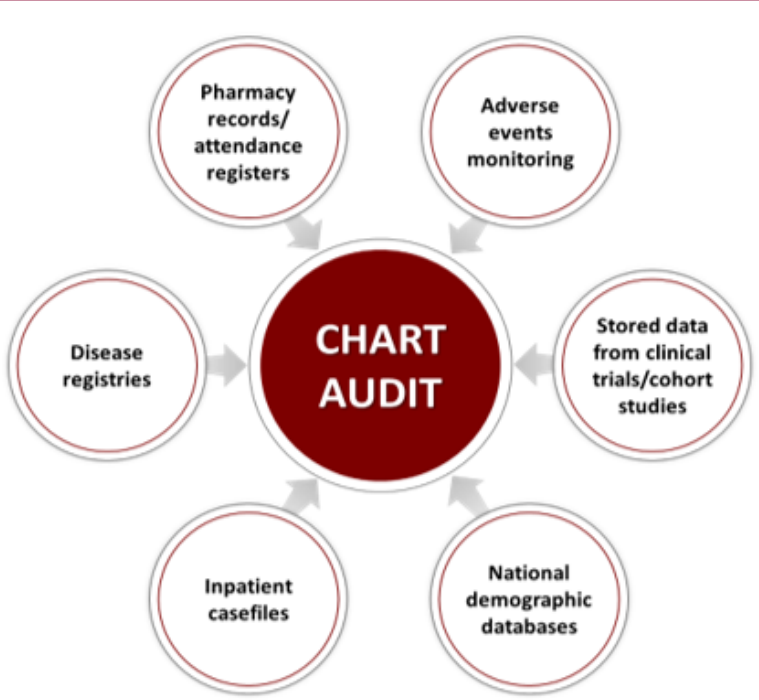

Figure 1: Different data sources of a chart audit.

\section{General Steps in Chart Audits}

a) Choose the therapeutic area: The first step is to assess the problem to audit to understand the costs, resources, or risks. Strong scientific evidence should be available to select the therapeutic area [8]. b) Identify the measures: Once a topic is selected, define exactly what is to be measured. The criteria must be outlined precisely, with specific guidelines [9].

c) Identify the patient population: Identify the accessible population based on the variables to assess the measure (Figure 2).

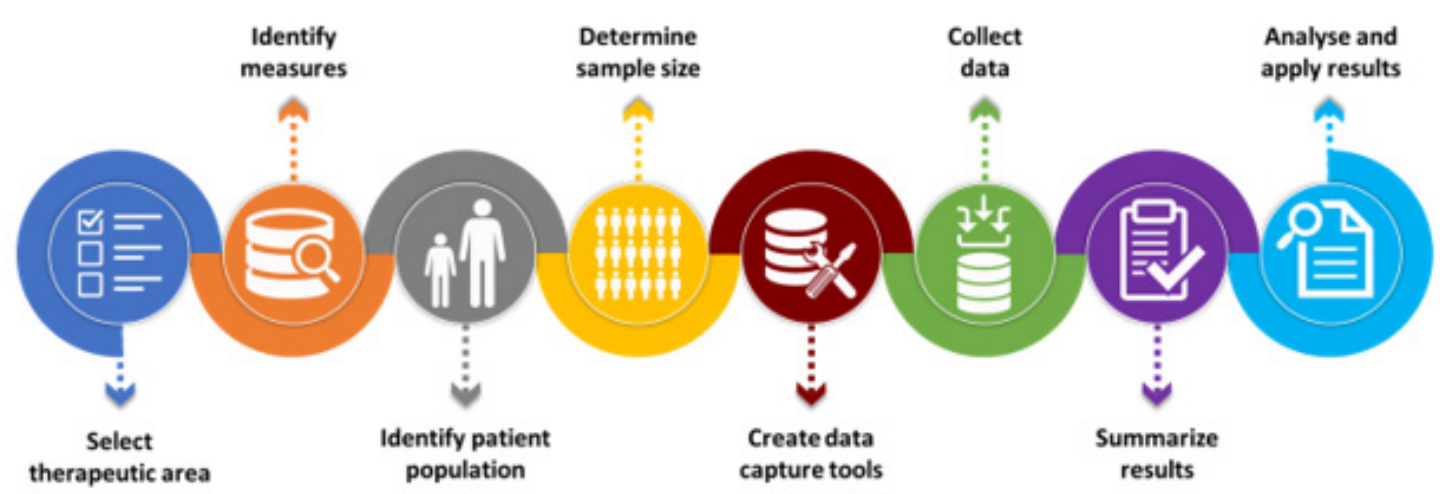

Figure 2: Chart audits - Essential steps. 
d) Determine the sample size: It is a critical step. Sample size calculations must account for all the available data, funding, support facilities, and ethics of the patients subject to research [10].

e) Develop the data capture tools: The electronic data capture system should be tailor made based on the essential variables related to the study outcome. This will increase the data accuracy and the study can be completed in time.

f) Data collection: The retrospective data (demographics, medical history, and treatment) is collected. The collected data can be quantitative or qualitative, and the data sources can be many.

g) Summarise the results: Summarising the data is a little more complex than just counting all the data sheets. The researcher must consider how the data will be used, and make sure the information is presented as per the study protocols [11].

h) Analyse and apply the results: Analyse the summarised data.

\section{How Chart Audits Contribute to Generate Business Insights?}

Chart audits usually play a major role in quality improvement. They can determine how frequently a specific treatment is offered or declined in a practice. If the treatment is not offered as suggested, then there are opportunities to improve the whole setup by identifying the crucial gaps [12]. 25\% scientific articles in clinical journals are contributed by the patient medical record data collected via retrospective chart audits [13]. Thus, they are a valuable tool to capture varied clinical information. Stakeholders working across varied patient populations striving to predict or prevent diseases, or promote health are likely to ask questions which would require data from medical records [14].

\section{Chart Audits are Usually Used to:}

a) Explore research questions often unanswered by prospective studies.

b) Explore questions in perinatal, neonatal, paediatrics, and mental health research due to the ethical implications involving vulnerable participants [15].

c) Study longitudinal disease pathogenesis over an extensive period.

d) Study quality assurance and improvement in clinical practice [15]

e) Provide data for future clinical research initiatives and hence identify unmet medical needs [16].

f) Establish a health history of patients who provide biologic samples for cutting-edge disease prediction research.

g) Generate real world evidence to perform economic analyses [12]

\section{How Beneficial is a Retrospective Chart Audit in Fulfilling Business Needs}

The use of data obtained from medical records via retrospective data collection has its advantages as well as limitations. The following are some:

\section{Advantages:}

a) Understanding the clinical characteristics of diseases-Chart audits help understand the characteristics associated with a disease or a health condition.

b) The prognosis of diseases over a follow-up periodInformation on the prognosis of the disease can be acquired from the development and advance notes of a patient over a stretch of time [17].

c) Characteristics of the patient population using a treatment service - Chart audits can also evaluate the attributes of patients part of a specific treatment service - inpatient or outpatient.

d) Safety in real world -The monitoring of safety events helps in supervising the health status and side effects of medications.

e) Observing health issues and indicators-Hospital and other records help identify the different indicators of patterns for various disorders.

f) Medical errors-Chart audits can be used to study medical errors for subsequent corrective and preventive actions [18].

\section{Limitations:}

a) Incomplete or missing data within the medical record.

b) Records lacking explicit patient information.

c) Difficulty in inferring or validating documented information.

d) Variability in the quality of documentation among health care personnel.

Thus, a robust process and a stepwise approach play a significant role when designing a patient chart audit. They can minimise the above limitations. For example, data collection can be done using a paper form or a classified electronic platform. The former is more cost effective and easier at the point of data collection. The data again needs to be transferred to an electronic tool for further analysis. Data collected directly on an electronic platform can make life easier for all users. It centralises the database and provides real time access to relevant data, which again can be cost effective for a large sample size. It also decreases data errors simultaneously [14]. Flowchart illustrating insights that can lead to informed decision (Figure 3).

\section{Ethical Considerations in Conducting A Patient Chart Audit}

Ethical Barriers in Conducting Patient Chart Audits Globally: There is very limited knowledge among researchers on the ethical concerns on retrospective data collection, especially when exempting informed consent. Researchers engage in discussions at various times for an answer when a request for informed consent exemption can suffice. Generally, any research on human subjects where data is collected in an un-anonymous 
format requires ethical protection [19]. Hence, it is advisable to approach an Ethics Committee (EC) for ethical sanction. A chart audit being a retrospective design of data collection that generally collects anonymized data and has limited scope for patient consent, can seek exemption. However, an approval is needed from the data privacy protection authority.

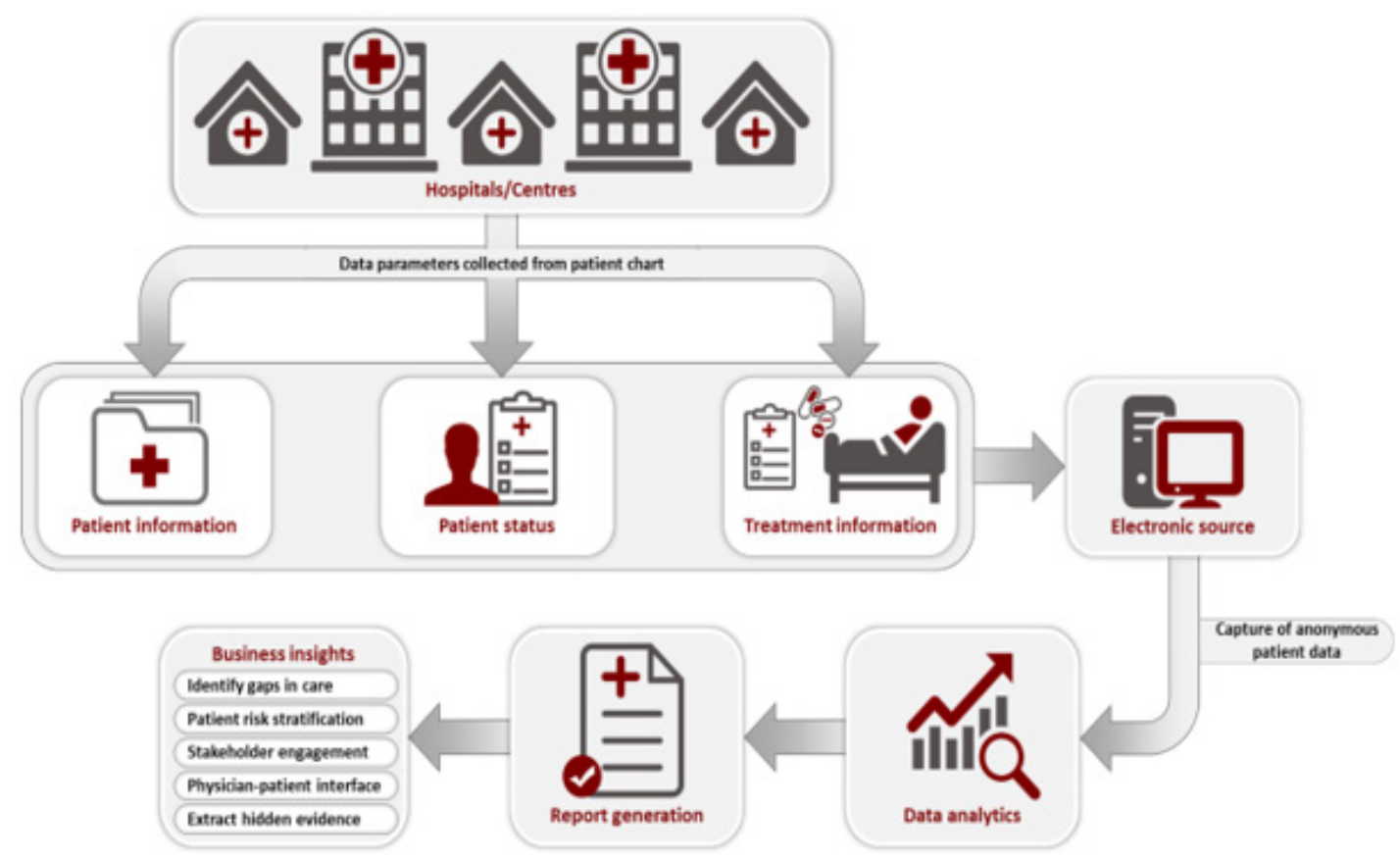

Figure 3: Flowchart illustrating insights that can lead to informed decision.

Chart Audits May, However, Involve Inputs from Patients at Different Levels

a) Patients may be asked to participate in surveys to determine if standards are met.

b) Patients can also be a part of individual chart projects when designing them, or can partake in complete programs, e.g., as members of steering groups [20].

\section{Chart Audit Projects Never Involve}

a) A completely new treatment or practice.

b) The use of control groups or placebo treatments.

c) Any disturbance to the patient beyond routine clinical management. [20].

d) Allocating patients randomly to different treatment groups

\section{Advisable Solutions to Overcome Barriers}

Data privacy and ethics in medical research is a very serious and highly regulated field [1]. In research involving the collection or study of existing data, documents, records, pathological or diagnostic specimens, if the information is recorded by the investigator in such a manner that subjects cannot be identified, directly or through identifiers linked to the subjects, then IRB approval is exempted according to 45CFR46 [21]. However this may vary depending on a country's individual regulatory guidelines because EC/
IRB members in many countries ensure that the anonymity and privacy protection of data is up held and it is also supported by the "International Ethical Guidelines for Biomedical Research Involving Human Subjects" [22]. According to international guidelines, ethics committees may waive the usual individual informed consent if the research involves minimal risk, and such consent would make the research impracticable. Evidence-based practices like chart audits which measure the impact of the reminder on patient outcomes (based on the review and confidential documentation of medical charts), may not require patient-level informed consent [23].

\section{Differentiating Retrospective Chart Audits (Real World Data) and Clinical Studies}

The debate on the battle between Randomised Clinical Trials (RCT) and Real-World Evidence (RWE) remains open for some despite the strengths of experimental and observational designs. Real world evidence and clinical trials are two different methods used by researchers to collect data and test hypothesis [24]. The basic differences between retrospective patient chart audit and clinical studies (RCTs) are summarised in the following table [25]. RCTs supply evidence for treatment effectiveness. Regulatory agencies use these studies to register a new drug. However, things become more complex when assessing the risks. RCTs do not provide ample safety data, do not use big enough population samples, and do not involve adequate follow ups to identify rare adverse effects. Also, the quality of the safety data identified in such studies is not always the greatest. Generalizability is also limited for RCTs 
because patients are at a high risk of adverse effects, are medically fragile, or have multiple comorbidities that are often excluded. A big amount of data from real world studies is often considered in the safety profile evaluation of a particular intervention to overcome these limitations [26] (Figure 4).

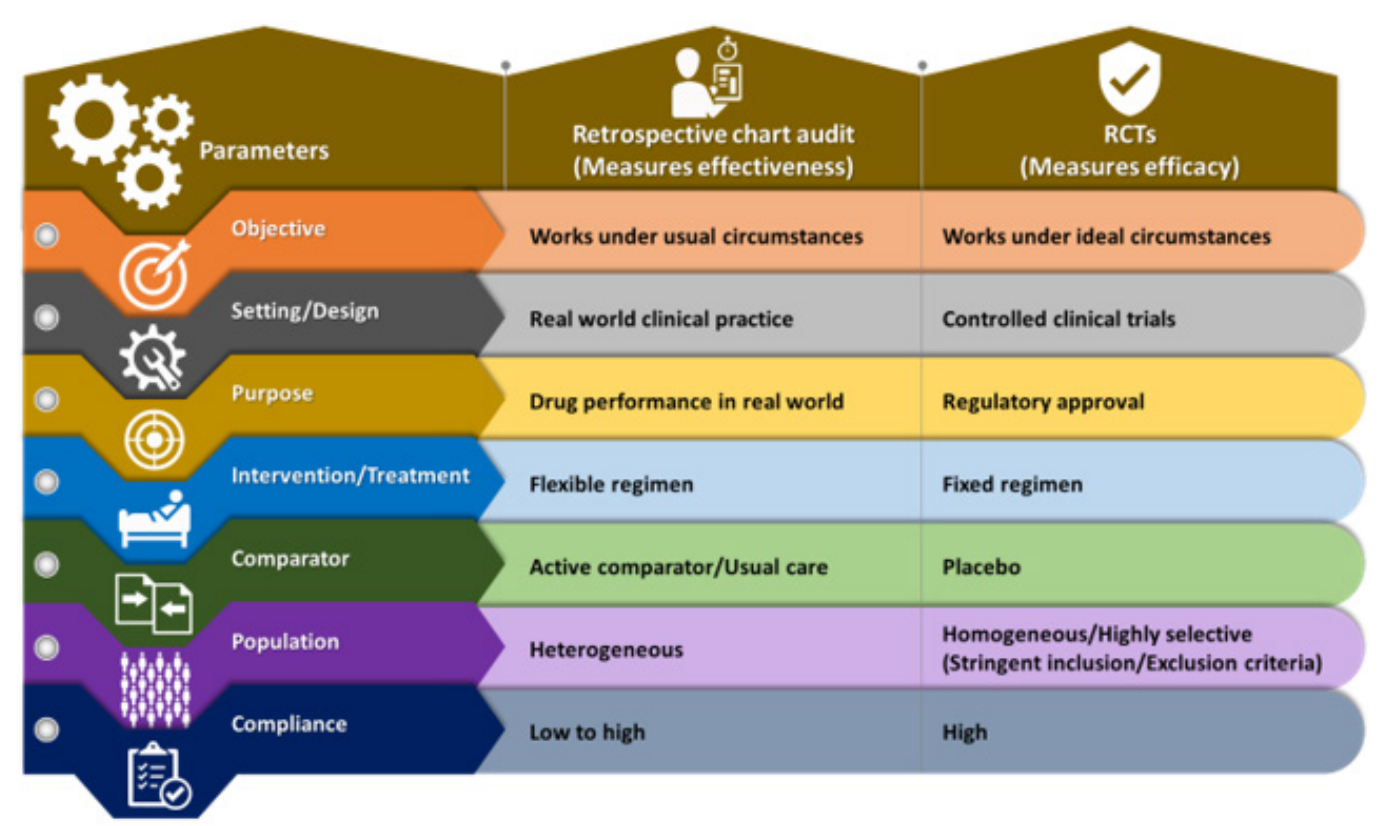

Figure 4: Differences between retrospective chart audit and RCTs.

\section{Conclusion}

The patient medical record has long been recognized as a rich source of information for conducting any type of clinical study. However, researchers must take a strategic approach to data collection and implement a rigorous methodology when conducting retrospective patient chart audits. Retrospective patient chart audit is an important methodology with vast advantages and can play a pivotal role in advancing healthcare management and advancing research. This type of study design uncovers hidden gems in the form of real-world data. The diverse stakeholders can use such studies to generalize results, understand the healthcare professional interests, assess the quality of data collected, and the level of accuracy in such studies. They can identify several latent patterns that were hither to unknown.

\section{References}

1. Vassar M, Holzmann M (2013) The retrospective chart review: important methodological considerations. Journal of Educational Evaluation for Health Professions 10(12): 1-7.

2. Gearing R, Mian I, Barber J, Ickowicz A (2006) A Methodology for Conducting Retrospective Chart Review Research in Child and Adolescent Psychiatry. J Can Acad Child Adolesc Psychiatry 15(3): 126134.

3. Worster A, Haines T (2004) Advanced statistics: Understanding Medical Record Review (MRR) Studies. Academic Emergency Medicine 11(2): 187-192.

4. (2017) EHR adoption rates: 20 must-see stats. Practice Fusion.

5. (2012) CGI Market Study of Electronic Medical Record (EMR) Systems in Europe.
6. (2017) The How's and Why's of Chart Audits.

7. Osborn G, Pike H, Smith M, Winter R, Vaughan-Williams E (2005) Quality of clinical case note entries: how good are we at achieving set standards? Annals of The Royal College of Surgeons of England 87(6): 458-460.

8. Esposito P, Canton A (2014) Clinical audit, a valuable tool to improve quality of care: General methodology and applications in nephrology. World Journal of Nephrology 3(4): 249-255.

9. (2017) A Practical Guide to Clinical Audit. $1^{\text {st }}$ (edn.); Quality and Patient Safety Directorate.

10. Gogtay N (2010) Principles of sample size calculation. Indian Journal of Ophthalmology 58(6): 517-518.

11. Kaprielian V, Gregory B, Sangvai D (2003) Chart Audits: The how's and why's p. 1-35.

12. Vijaykanth A (2017) Patient Chart Audit- A General Overview. Industry Insights.

13. Gilbert E, Lowenstein S, Koziol-McLain J, Barta D, Steiner J (1996) Chart Reviews In Emergency Medicine Research: Where Are The Methods? Annals of Emergency Medicine 27(3): 305-308.

14. Gregory K, Radovinsky L (2012) Research strategies that result in optimal data collection from the patient medical record. Applied Nursing Research 25(2): 108-116.

15. Colón-Emeric C, Schenck A, Gorospe J, McArdle J, Dobson L, et al. (2006) Translating vidence-Based Falls Prevention into Clinical Practice in Nursing Facilities: Results and Lessons from a Quality Improvement Collaborative. Journal of American Geriatric Society 54(9): 1414-1418.

16. Pronovost P, Berenholtz S, Ngo K, McDowell M, Holzmueller C, et al. (2003) Developing and pilot testing quality indicators in the intensive care unit. Journal of the Critical Care 18(3): 145-155.

17. Allison J, Wall T, Spettell C, Calhoun J, Fargason C, et al. (2000) The Art and Science of Chart Review. Journal on Quality Improvement 26(3): 115-136. 
18. Sarkar S, Seshadri D (2014) Conducting Record Review Studies in Clinical Practice. Journal of Clinical and Diagnostic Research 8(9): 1-4.

19. Schroter S, Plowman R, Hutchings A, Gonzalez A (2006) Reporting ethics committee approval and patient consent by study design in five genera medical journals. Journal of Medical Ethics 32(12): 718-723.

20. (2009) How To: Apply Ethics to Clinical Audit. $3^{\text {rd }}$ (edn.); Bristol: University Hospitals Bristol, NHS.

21. (2009) Protection of Human Subjects: Title 45, Code of Federal Regulations. US Department of Human Health and Services.

22. (2002) International Ethical Guidelines for Biomedical Research

ISSN: 2574-1241

DOI: 10.26717/BJSTR.2018.07.001476

Uttam Barick. Biomed J Sci \& Tech Res

(C) (i) This work is licensed under Creative

Submission Link: https://biomedres.us/submit-manuscript.php
Involving Human Subjects. Council for International Organizations of Medical Sciences.

23. (2013) WHO. Ethical issues in Patient Safety Research Interpreting existing guidance. Geneva: WHO p. 1-41.

24. (2017) Difference between Observational Studies and Clinical Trials. Observational Studies vs Clinical Trials.

25. Barick U (2017) Unraveling the Potential of Real-World Studies.

26. Trotta F (2012) Discrepancies between observational studies and randomized controlled trials. Pharmaco Vigilance 73(11): 1.

Assets of Publishing with us
BIOMEDICAL
RESEARCHES

